\title{
Research of Defogging base on Wavelet Transforms
}

\author{
Nie Ning ${ }^{1}, \mathrm{Wu} \mathrm{SiJiu}^{2}$, Cheng WeiDong ${ }^{3}$ \\ ${ }^{1)}$ Department of Electronic Engineering, Chengdu University of Information Technology, Chengdu. (422181769@qq.com) \\ 2) The Information Center, Chengdu University of Information Technology, Chengdu. (wsj@ .cuit.edu.cn) \\ ${ }^{3)}$ Department of Electronic Engineering, Chengdu University of Information Technology, Chengdu. (chengwd@cuit.edu.cn)
}

\begin{abstract}
This paper are compared several common methods of single image defogging. And this paper introduced a improving fast defogging method of wavelet decomposition based on the dark color prior. The results prove that this method can effectively improve dark color prior. The defogged image is more colorful. It greatly improves the computing speed.
\end{abstract}

Keywords — defogging, image, Dark color prior, Wavelet transform, quickly

\section{基于小波变换的图像去雾研究}

\author{
聂宁 $^{1} \quad$ 吴四九 $^{2}$ 程卫东 $^{3}$ \\ 1) 成都信息工程学院电子工程学院, 成都, 四川, 中国 \\ 2) 成都信息工程学院信息中心, 成都, 四川, 中国 \\ 3) 成都信息工程学院电子工程学院, 成都, 四川, 中国
}

摘 要 本文通过对比分析常用的几种图像去雾方法, 并在此基础上进行研究, 提出了利用小波分解的基于暗原色先验去雾的 改进算法。实验结果显示, 该改进的算法可以有效改进暗原色先验算法的不足, 去雾后的图像色彩丰富, 而且可以有效的减少运行 时间, 提高运行速度。

关键词 去雾, 图像, 暗原色先验, 小波变换, 快速

\section{1. 引言}

雾霊天气是一种常见的天气情况, 在雾天条件下, 大 气中的杂质会使能见度降低, 光线会有一定程度上的衰减, 由于杂质散射和反射影响, 户外采集到的图像对比度和亮 度、分辨率等都会严重下降, 这给监测、跟踪、图像处理 等方面带来了极大困难, 因此, 在雾天对图像进行去雾增 强有着很重要的现实意义。

早期的图像去雾主要是利用多幅图像对比, 从而得到 景深, 大气能见度等一些基本参数 ${ }^{[1]}$, 然后根据这些参数 推导还原去雾图像, 但这种方法对单幅图像去雾不太适用。 现阶段对单幅图像去雾主要有两类方法: 一类是将图像对 比度增强, 主要方法有直方图均衡化、同态滤波。文献[2] 提出用直方图均衡化处理雾天图像, 这种方法对薄雾天气 情况下的图像可以有些改善, 但是浓雾情况下效果很差, 会有失真情况出现。祝培等人[3]对直方图均衡化进行了改 进, 对天空区域用正态分布进行求解, 然后用移动模板对
局部区域进行直方图均衡。文献[4]提出用同态滤波来进行 去雾, 他将图像有雾部分看成低频部分, 要恢复的区域看 成高频部分, 分离出图像的高频和低频, 通过降低低频, 增强高频来进行去雾, 这种方法没有考虑到图像的深度, 去雾效果有限。另一类是根据雾天降质图像的形成原理, 结合大气散射物理模型进行去雾。主要方法有暗原色先验 去雾。何凯明等人提出基于暗原色先验去雾方法 ${ }^{[5]}$, 该方 法通过对 500 多幅无雾图像进行分析, 得出所有图像都存 在暗原色区域, 并且这些暗原色区域像素值为零。有雾区 域的像素被雾所填充, 通过对该区域分析可以估计环境光 和大气光, 从而恢复出原图像。

\section{2. 常见的各种去雾效果比较}

基于物理模型和非物理模型在去雾思路上大为不同, 非物理模型本质上是增强图像的对比度和对图像的颜色进 行较正, 它并没有针对雾天图像的形成机理进行有效补偿, 因此去雾效果有限。而物理模型是基于大气物理散射的规 
律建立图像还原模型, 但要知道大气光和环境光, 场景深 度、空气折射率等参数 ${ }^{[8]}$ 。非物理模型去雾主要有直方图 均衡化和同态滤波方法, 基于物理模型去雾的主要是暗原 色先验去雾。

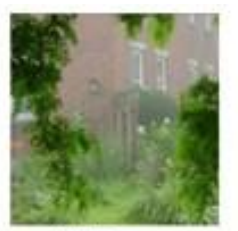

(a) 原国侻

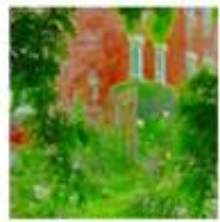

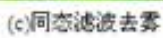

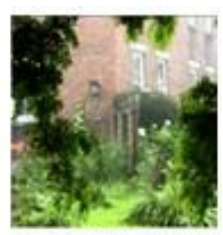

(b)直方圆均得化图

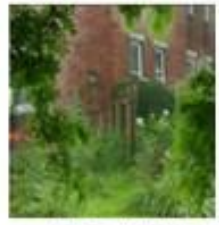

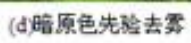

图 1 常见的去雾方法比较

通过实验对比可以看到直方图均衡化方法对于薄雾天 气下的图像恢复还是不错的, 细节等方面也比较接近现实, 但是在浓雾条件下, 图像大部分显示为灰色, 在这种情况 下直方图均衡化就不适用, 而且用直方图均衡化时, 图像 灰度拉伸到整个图像, 图像经过直方图均衡化以后出现明 显的饱和失真。同态滤波方法对于薄雾图像恢复也可以, 图像中有雾部分变化比较缓慢, 可以看成是图像的低频部 分，通过降低低频成分，增强高频成分可以达到去雾效果。 但是浓雾条件下它的效果很差, 许多情况下图像过于饱和, 有些区域会出现图像失真, 而暗原色先验去雾后的图像色 彩比较真实, 细节比较真实, 整体上来说比较令人满意。

但其运算速度太慢, 该方法最大的问题在于对透射率 $\mathrm{t}$ 的计算并优化。在计算图像的暗原色区域时需要对图像的 每一个像素点进行比较, 该方法比较耗时。而且在具体实 验过程中, 通过对每个相素对比得到暗原色区域, 运算量 较大, 因此本文将对暗原色先验去雾和小波变换进行简单 介绍, 并结合小波分解和暗原色先验对图像进行快速去雾。

\section{3. 暗原色先验去雾}

\section{1 大气散射物理模型}

根据文献[6]提出的大气物理散射模型可知, 雾天图像 可表示为:

$$
I(\mathrm{x})=\mathrm{J}(\mathrm{x}) \mathrm{t}(\mathrm{x})+A(\mathrm{x})(1-t(\mathrm{x}))
$$

$I(\mathrm{x})$ 指平时拍摄到的有雾图像, $\mathrm{J}(x)$ 为要得到的无雾 图像, $A(\mathrm{x})$ 环境光成分, $t(\mathrm{x})$ 是透射率, 图像去雾就是要
从 $I(\mathrm{x})$ 中得到无雾图像 $\mathrm{J}(\mathrm{x})$, 环境光 $A(x)$, 透射率 $t(\mathrm{x})$ 。 方程中的第一项 $\mathrm{J}(\mathrm{x}) \mathrm{t}$ (为直接衰减项, 第二项 $A(\mathrm{x})(-1 t$ （徥大气光成分。

\section{2 暗原色先验}

何凯明通过对 500 多幅无雾图像进行观察实验对比得 到: 大数多无雾图像在其 $(\mathrm{R}, \mathrm{G}, \mathrm{B})$ 三个通道至少有一个颜色 通道具有像素值很低的区域, 也就是说, 该区域光的强度 值是一个很小的数, 对于图像 $\mathrm{J}$, 定义为:

$$
J^{\text {dark }}(\mathrm{x})=\min _{c \in\{r, g, b\}}\left(\min _{y \in \Omega(\mathrm{x})}\left(\mathbf{J}^{c}(\mathrm{y})\right)\right)
$$

$J^{c}$ 是 $J$ 的颜色通道, $\Omega(\mathrm{x})$ 是暗原色区域, 研究表明, 图像的暗原色区域强度接近为 0 。上式中 $\mathrm{J}$ 代表无雾图像, $J^{d a r k}$ 是无雾图像 $J$ 的暗原色, 将以上图像颜色通道存在低 像素区域的规律统称为暗原色先验。

\section{3 暗原色去雾算法}

因为任何图像都是要受到周围环境影响的, 其大气光 $\mathrm{A}$ 总是大于 0 的, 并且同一区域的透射率基本相同, 我们 对 (1) 式两边同时取最小值, 可得:

$$
\min _{y \in \Omega(\mathrm{x})}\left(\frac{I^{c}(\mathrm{y})}{A^{c}}\right)=\tilde{t}(\tilde{\mathrm{x}}) \min _{y \in \Omega(\mathrm{x})}\left(\frac{J^{c}(\mathrm{y})}{A^{c}}\right)+(1-\tilde{t}(\tilde{\mathrm{x}}))
$$

对 RG,B 三通道进行最小值运算，可得：

$$
\min _{c}\left(\min _{y \in \Omega(\mathrm{x})}\left(\frac{I^{c}(\mathrm{y})}{A^{c}}\right)\right)=\tilde{t}(\tilde{\mathrm{x}}) \min _{c}\left(\min _{y \in \Omega(\mathrm{x})}\left(\frac{J^{c}(\mathrm{y})}{A^{c}}\right)\right)+(1-\tilde{t}(\tilde{\mathrm{x}}))
$$

又因为无雾图像的暗原色趋近于 0 , 即:

$$
J^{\text {dark }}(\mathrm{x})=\min _{c}\left(\min _{y \in \Omega(\mathrm{x})}\left(\mathrm{J}^{c}(\mathrm{y})\right)\right)=0
$$

又因为 $A^{c}$ 总为正数, 故得出

$$
\min _{c}\left(\min _{y \in \Omega(\mathrm{x})}\left(\frac{J^{c}(\mathrm{y})}{A^{c}}\right)=0\right.
$$

将（6）式 代入（4）中, 可得到透射率和 $\mathrm{t}$ :

$$
\tilde{t}(\tilde{\mathrm{x}})=1-\min _{c}\left(\min _{y \in \Omega(\mathrm{x})}\left(\frac{I^{c}(\mathrm{y})}{A^{c}}\right)\right)
$$

在去雾过程中, 完全去雾会使图像看起来不是很真实, 而且会失会深度感, 因此在上式中引入一个常数 $\omega(0<\omega<1$, 保留部分远处的雾, 上式变为: 


$$
\tilde{t} \tilde{\mathrm{x}})=1-\omega \min _{c}\left(\min _{y \in \Omega(\mathrm{x})}\left(\frac{I^{c}(\mathrm{y})}{A^{c}}\right)\right)
$$

通过上式我们可以得到折射率的基本估算方法, 但是 它不是很精确, 何凯明在其文献中用了软抠图来求图像的 透射率分布函数 $t(\mathrm{x})$, 通过解下列方程可得到透射率 $\mathrm{t}$.

$$
(L+\lambda U) t=\lambda \tilde{t}
$$

$\lambda$ 为引入的参数, $L$ 是拉普拉斯描图矩阵, 算出透射 率 $t(\mathrm{x})$ 以后, 只要再估算出大气光 $\mathrm{A}$ 的值, 将透射率和 $\mathrm{A}$ 值代入(1)式中就可恢复出 $J(\mathrm{x})$ :

$$
J(\mathrm{x})=\frac{I(\mathrm{x})-\mathrm{A}}{\max \left(\mathrm{t}(\mathrm{x}), \mathrm{t}_{0}\right)}+A
$$

大气光 $\mathrm{A}$ 的估计方法: 在原图像中, 将各像素点亮度 递减排列, 取前 $0.1 \%$ 的点所在位置处的最大值作为 $\mathrm{A}$ 值。

\section{4 图像小波分解}

对有雾图像来说, 有雾部分色调变化比较平缓, 纹理 结构比较简单, 可以将雾影响部分看成图像低频部分, 为 很好的得到雾影响部分区域, 我们可以对图像进行小波变 换, 图像经过小波变换后, 可通过在分解的低频图像上, 识别出雾区域, 并对图像雾区的低频和高频适当处理。采 用 Mallat 算法进行小波分解 ${ }^{[7]}$, 并将其原理表述如下:

定义三个 “二维小波” 如式 (11) 所示, 其中 $v_{j}^{2}(\mathrm{j} \in Z)$ 是 $\mathrm{L}^{2}\left(\mathrm{R}^{2}\right)$ 的可分离多分辨分析, 并且令 $\phi(\mathrm{x}, \mathrm{y})=\phi(\mathrm{x}) \phi(\mathrm{y})$ 是对应二维尺度函数, 而 $\varphi(\mathrm{x})$ 是与尺 度函数对应的一维标准正交小波。

$$
\left\{\begin{array}{l}
\varphi^{1}(\mathrm{x}, \mathrm{y})=\phi(\mathrm{x}) \varphi(\mathrm{y}) \\
\varphi^{2}(\mathrm{x}, \mathrm{y})=\varphi(\mathrm{x}) \phi(\mathrm{y}) \\
\varphi^{3}(\mathrm{x}, \mathrm{y})=\varphi(\mathrm{x}) \varphi(\mathrm{y})
\end{array}\right.
$$

则:

$$
\left\{\begin{array}{l}
2^{-j} \varphi^{1}\left(2^{-j} x-m, 2^{-j} y-n\right) \\
2^{-j} \varphi^{2}\left(2^{-j} x-m, 2^{-j} y-n\right) \\
2^{-j} \varphi^{3}\left(2^{-j} x-m, 2^{-j} y-n\right)
\end{array}\right.
$$

分别是 $L^{2}\left(\mathrm{R}^{2}\right)$ 内的标准正交基。设 $f=f(\mathrm{x}, \mathrm{y}) \in \mathrm{v}_{j}^{2}$ 为待分析图像信号, 其二维逼近图像为:

$$
A_{j} f=A_{j+1} f+D_{j+1}^{1} f+D_{j+1}^{2} f+D_{j+1}^{3} f
$$

$$
\begin{aligned}
& A_{j+1} f=\sum_{m=-\infty}^{\infty} \sum_{n=-\infty}^{\infty} C_{j+1}(\mathrm{~m}, \mathrm{n}) \phi_{j+1}(\mathrm{~m}, \mathrm{n}) \\
& D_{j+1}^{i} f=\sum_{m=-\infty}^{\infty} \sum_{n=-\infty}^{\infty} D_{j+1}^{i}(\mathrm{~m}, \mathrm{n}) \phi_{j+1}(\mathrm{~m}, \mathrm{n}), i=1,2,3
\end{aligned}
$$

根据小波函数和尺度变换正交性, 由(11) (12) (13)可 得:

$$
\begin{aligned}
& C_{j+1}(\mathrm{~m}, \mathrm{n})=\sum_{k=-\infty}^{\infty} \sum_{l=-\infty}^{\infty} h(\mathrm{k}-2 \mathrm{~m}) \mathrm{h}(\mathrm{l}-2 \mathrm{n}) C_{j}(k, l) \\
& \left\{\begin{array}{l}
D_{j+1}^{1}(\mathrm{~m}, \mathrm{n})=\sum_{k=-\infty}^{\infty} \sum_{l=-\infty}^{\infty} h(\mathrm{k}-2 \mathrm{~m}) g(1-2 \mathrm{n}) C_{j}(k, l) \\
D_{j+1}^{2}(\mathrm{~m}, \mathrm{n})=\sum_{k=-\infty}^{\infty} \sum_{l=-\infty}^{\infty} \mathrm{g}(\mathrm{k}-2 \mathrm{~m}) \mathrm{h}(\mathrm{l}-2 \mathrm{n}) C_{j}(k, l) \\
D_{j+1}^{3}(\mathrm{~m}, \mathrm{n})=\sum_{k=-\infty}^{\infty} \sum_{l=-\infty}^{\infty} \mathrm{g}(\mathrm{k}-2 \mathrm{~m}) g(1-2 \mathrm{n}) C_{j}(k, l)
\end{array}\right.
\end{aligned}
$$

引入矩阵算子,二维 Mallat 分解算法为:

$$
\left\{\begin{array}{l}
C_{j+1}=H_{r} H_{c} C_{j} \\
D_{j+1}^{1}=H_{r} G_{c} C_{j} \\
D_{j+1}^{2}=G_{r} H_{c} C_{j} \\
D_{j+1}^{3}=G_{r} G_{c} C_{j}
\end{array}, j=0,1,2 \cdots, J\right.
$$

二维 Mallat 小波分解算法结构框图如图 2 所示, 从图 中可以看出, 小波变换就是把图像信号分解成多个低频子 带分量, 并且每一层小波分解都将上一层图像分解成 4 个 子带图像, 即, 低频信息子图 (亮度分量图像) 和三幅高 频子图 (水平边缘、垂直边缘和对角线边缘子图)。这样逐 级分解就会形成塔形分解结构。图 3 是图像三级小波分解 示意图。通过该金字塔结构能很好地将表示图像内容的低 频信息与表示图像细节的高频信息分离出。因此, 小波变 换能用不同的方法, 并且在不同的尺度上增强不同频率范 围内图像的细节分量。

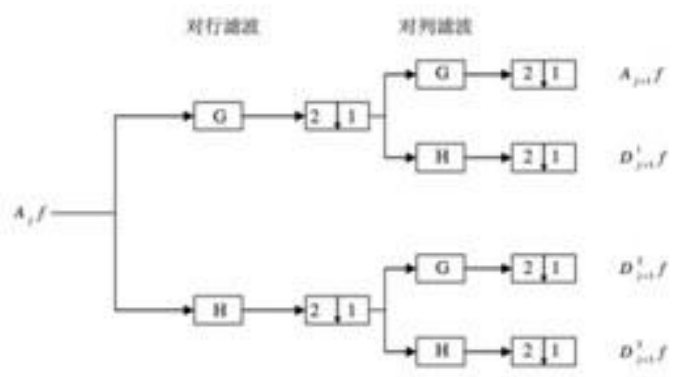

图 2 二维 Mallat 小波分解算法示意图 


\begin{tabular}{|c|c|c|c|}
\hline LL3 & HL3 & \multirow{2}{*}{ HL2 } & \multirow{3}{*}{ HL1 } \\
LH3 & HH3 & \\
\cline { 1 - 1 } LH2 & HH2 & \\
\cline { 1 - 1 } & & \\
& & \\
& HH1 & HH1 \\
& & \\
\hline
\end{tabular}

图 3 图像三级小波分解示意图

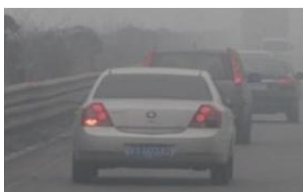

(a)原图

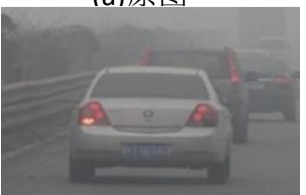

(a)原图

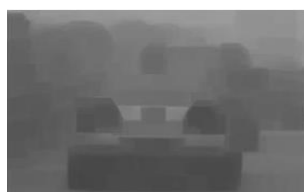

(b) 暗原色

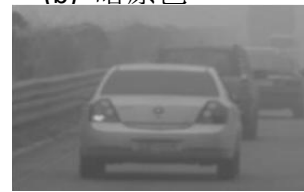

(e)小波分解

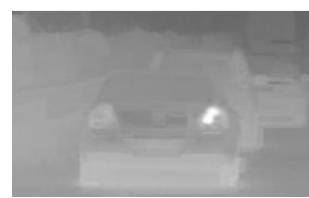

(c) 透射图像

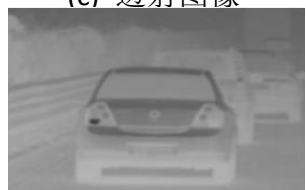

(f)高频透射

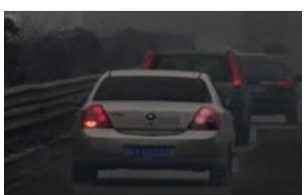

(d)暗原色去雾

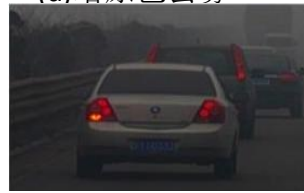

(g)本文去雾

图 4 图像大小为 $320 * 240$ 的去雾比较

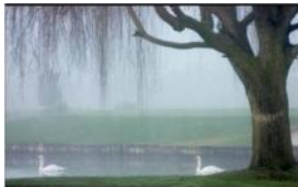

(a)原图 2

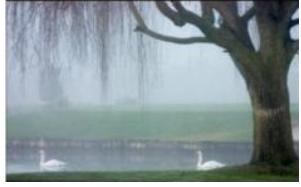

(a)原图 2

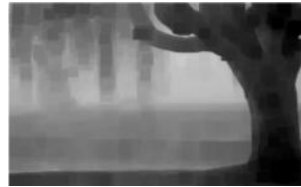

(b) 暗原色

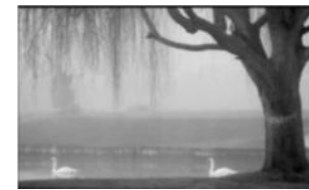

(e) 小波分解

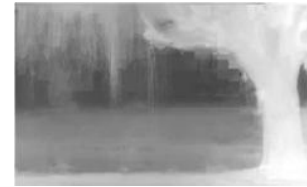

(c)透射图像

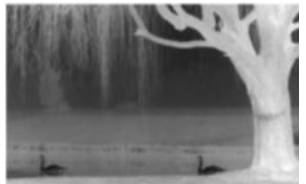

(f)高频透射

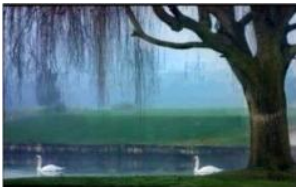

(d)暗原色去雾

图 5 图像大小为 $640 * 480$ 的去雾比较

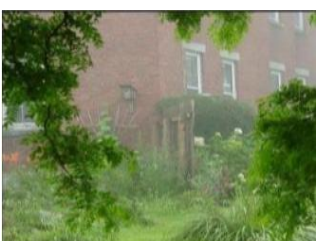

(a)原图 3

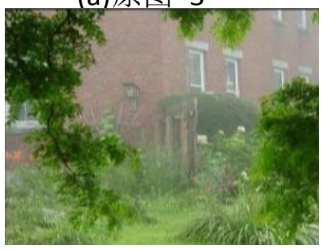

(a)原图 3

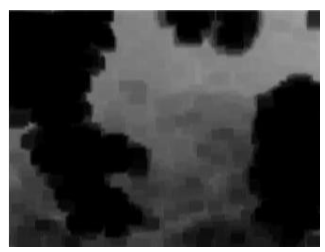

(b)暗原色

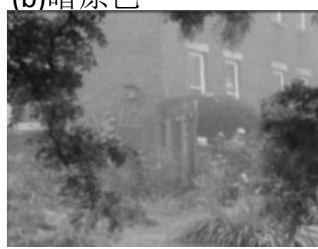

(e)小波分解

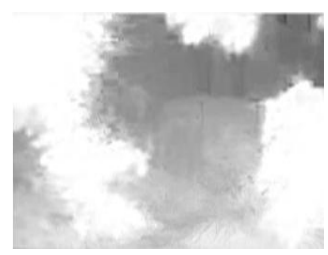

(c)高频透射

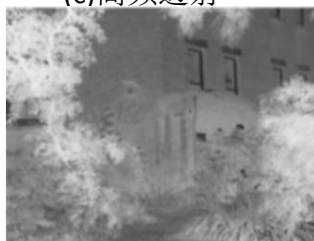

(f)高频透射

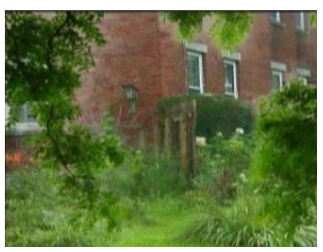

(d)暗原色去雾

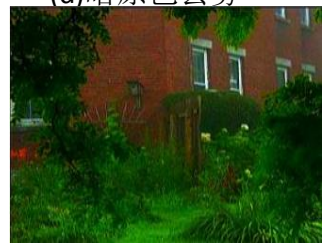

(g)本文方法

图 6 图像大小为 $800 * 600$ 的去雾比较 
在实验中我们分别将三组图在操作系统为 Windows $\mathrm{XP}, \mathrm{CPU}$ 为酷睿 2 双核 $3.30 \mathrm{GHZ}$, 内存 $8 \mathrm{~GB}$ RAM 的 PC 上 进行测试。所用时间如下表所示:

表 1 算法处理时间对比

\begin{tabular}{|c|c|c|c|}
\hline \multicolumn{4}{|c|}{ 暗原色先验去雾和本文所用方法相比较 (单位:s ) } \\
\hline 图像大小 & $320 * 240$ & $640 * 480$ & $800 * 600$ \\
\hline 暗原色先验去雾 & 13.045231 & 28.545893 & 37.050698 \\
\hline 本文所用方法 & 0.519559 & 1.543746 & 2.760709 \\
\hline
\end{tabular}

4. 结论

从以上图像对比可以看出, 利用小波变换的多分辨率 分解可以有效区分图像的低频和高频部分, 将图像中含雾 的低频分离出来, 得到图像的高频透射区域图像, 对图像 低频进行滤波的同时，对图像高频透射区域进行增强。基 于小波分解的暗原色先验去雾算法比文献[5]去雾效果更好 明显, 图像细节更加丰富, 色彩更加真实, 在图片的清晰 度和运算速度有了很大的改进。

本文算法的主要优点是在速度快、方法简单的条件下 取得了较好的去雾效果。但是算法本身也存在一定的不足, 如在估计环境光时的精确度偏低以及对浓雾情况下恢复的 图像效果欠妥, 小波分解所采用的层数过多过少都会影响 后期去雾效果, 今后将进一步改进算法, 以寻找更有效更 适合的模型和参数使其效果更好。

\section{参考文献(References)}

[1] Oakly J P and Satherley B L, "Improving image quality in poor visibility condition using a physical model for contrast degradation". IEEE Transactions On Image Processing, vol. 2, no. 7, pp.167-179. 1998

[2] oung-Youn Kim, lee-Sup Kim and Seung-Ho Hwang, "An advanced Contrast Enhancement using partially overlapped Sub-Block Histogram Equalization.” IEEE Transactions on Circuits and systems for Video Technology,pp.475-484,2001

[3] Zhu Pei, Zhu Hong, Qian Xue ming. "An image clear mess method for fog." Normal of image and graphics, vol.1, pp. 126-130. September 2004

[4] Chen Gong, Wang Tang, Zhou Heqin, "A novel physics-based method for restoration of foggy day images". Joumal of image and graphics, pp.888-893, 2008

[5] He kai ming, Sun Jian, TANG Xiao ou. "Single image haze removal using dark charnel prior". Proceedings of IEEE Conference on Computer Vision and Pattern Recognition (CVPR). Miami, FL, USA: IEEE Computer Society, 2009, pp, 1956-1963.

[6] Mc Cartney E J. "Optics of Atmosphere: scattering by Molecules and Particles", New York: John Wiley and Sons, pp.23-32,1996

[7] Mallat S . A theory for multiresolution signal decomposition: The wavelet representation. IEEE Trans, PAMI, vol. 7, no. 11,pp, 674-693.1989

[8] Narasimhan S G and S K Chromatic "Framework for Vision in Bad Weather". In Proceedings of the IEEE Conference on Computer Vision and Pattern Recognition 2000. 